\title{
BRIGHT NEW START FOR EUROPE?
}

The impending launch of the European $\mathrm{X}$-Ray Free-Electron Laser (European XFEL) at Hamburg in Germany in early 2017 is cause for excitement for anyone interested in the structure of matter. The boon for chemistry and molecular biology is that this new source of ultrashort pulses of $\mathrm{X}$-rays with unprecedented brightness could make it possible to perform detailed structural studies on noncrystalline materials and perhaps on single molecules: to enable routine diffraction without crystals.

What's more, the femtosecond pulses of the European XFEL - which allow structures to be determined in a single shot before the samples are destroyed through extreme ionization - repeat at a much higher frequency than those of existing freeelectron lasers: 27,000 times a second, compared with 120 per second at the Linac Coherent Light Source (LCLS) of the SLAC National Accelerator Laboratory in Stanford, California. This will enable data collection times to be much shorter, and will facilitate the collection of dynamical snapshots at the atomic scale.

The opportunities for materials science have already been amply illustrated by existing XFELs such as the LCLS and FLASH, located at the same Hamburg site as the European XFEL. These facilities are themselves an evolution of synchrotron sources of X-rays, such as the Diamond Light Source in the UK and PETRA III in Hamburg, which were in turn an outgrowth of particle physics. Such ultrabright $\mathrm{X}$-ray beams make it possible to perform diffraction on very small samples, but it was only in 2000 that the potential of then-nascent XFELs became clear with a theoretical demonstration that structure determination could potentially outrun beam damage ${ }^{1}$.

The value for materials science was made explicit when this possibility was confirmed experimentally in 2006. Researchers using FLASH in Hamburg reported diffraction patterns from a nanopatterned non-periodic structure in a film of silicon nitride, allowing the structure to be reconstructed from the scattering that occurred before the sample was destroyed ${ }^{2}$.

Many subsequent studies have confirmed the value of bright X-ray sources for investigating the structure of materials. For example, Fuchs et al. have recently used synchrotron sources in Germany to develop a non-destructive tomographic technique using extreme ultraviolet and soft X-rays that can map deeply buried layers with a resolution of a few nanometres ${ }^{3}$.

Dynamical processes can be explored too. For example, Gaudin et al. have shown that it should be feasible with XFELs to obtain simultaneous, ultrafast (subpicosecond) structural and electronic information on samples using X-ray absorption spectroscopy. They demonstrate the idea for molybdenum using the LCLS 4 .

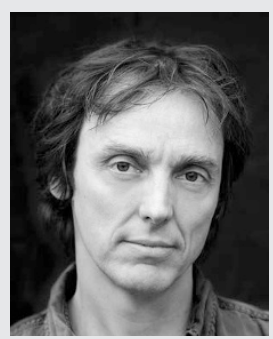

PHILIP BALL

The European XFEL promises to open up new avenues of study, for example the investigation of matter under extreme conditions of density and energy density, relevant to understanding planetary interiors ${ }^{5}$. Already, using X-ray scattering from LCLS, Brown et al. report that shock compression and melting of carbon shows signs of creating a glassy plasma phase possibly relevant for the interiors of Uranus, Neptune and white dwarfs ${ }^{6}$. One of the instruments on the European XFEL is dedicated to exploring this kind of high-energydensity environment, opening up the expanding new frontier of warm dense matter ${ }^{7}$.

References

1. Neutze, R., Wouts, R., van der Spoel, D., Weckert, E. \& Hajdu, J. Nature 406, 752-757 (2000).

2. Chapman, H. N. et al. Nature Phys. 2, 839-843 (2006).

3. Fuchs, S. et al. Sci. Rep. 6, 20658 (2016).

4. Gaudin, J. et al. Sci. Rep. 4, 4724 (2014).

5. Appel, K. et al. Plasma Phys. Controlled Fusion 57, 014003 (2015)

6. Brown, C. R. D. et al. Sci. Rep. 4, 5214 (2014).

7. Valenza, R. A. \& Seidler, G. T. Phys. Rev. B 93, 115135 (2016).

Drug-loaded liposomes functionalized with proteins from the cell membrane of leukocytes target inflamed tissues without eliciting a substantial immune response.

\section{Kenneth A. Dawson and Yan Yan}

E: arly successful applications of nanoparticles for drug delivery have typically exploited passive accumulation effects. Nanocarriers for active targeting have, however, been slower to deliver clinical success. One reason is that sophisticated biological recognition mechanisms identify synthetic nanoconstructs as foreign to the organism ${ }^{1}$ and clear them, thereby preventing access to the target. This, and other challenges, are especially relevant for nanocarriers made by conventional nanoparticle-synthesis procedures ${ }^{2}$.

An alternative strategy to bottom-up synthesis is to 'borrow' some of the 